\title{
Aspartate aminotransferase-platelet ratio index and body mass index in children with fatty liver
}

\author{
Ninung RD Kusumawati, Maria Mexitalia, Suci Romadhona
}

\begin{abstract}
Background Obesity in children is becoming a global epidemic. Non-alcoholic fatty liver disease (NAFLD) is a highly prevalent and potentially serious complication of childhood obesity. The early identification of fibrosis is important in children with NAFLD in order to prevent the development of liver disease in adulthood. One non-invasive procedure to predict liver fibrosis is the aspartate aminotransferase (AST)-platelet ratio index (APRI).

Objective The purpose of our study was to assess a correlation between APRI and body mass index (BMI) in obese children with fatty liver.

Methods A cross-sectional study was conducted from August to September 2007. Subjects were obese children from one junior high school in Semarang. Complete blood count, transaminase enzyme measurement, and abdominal ultrasound (USG) were performed on each subject. Only subjects with bright liver on USG underwent APRI analysis. Spearman's correlation was used for statistical analysis.

Results Of 37 obese children, 19 children had bright liver on USG. Their mean APRI was 0.16 (SD 0.119). Only one obese subject $(1 / 37)$ with bright liver had an APRI $>0.5$. APRI was significantly correlated to alanine amino transferase (ALT) levels $(r=0.62)$, but not significantly correlated to weight and BMI.

Conclusion There was no correlation between APRI and BMI. Only 1 out of 37 obese children with fatty liver had APRI levels indicating the presence of liver fibrosis. [Paediatr Indones. 2012;52:181-4].
\end{abstract}

Keywords: APRI, fatty liver, liver fibrosis, obese children

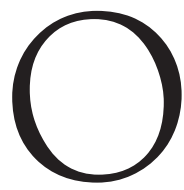
besity is becoming a global epidemic in children, creating a double burden of nutritional problems in developing nations such as Indonesia. Adiposity affects almost all organs of the body and may lead to NAFLD. ${ }^{1}$ NAFLD is a highly prevalent and potentially serious complication of childhood obesity. ${ }^{2}$ NAFLD is a hepatic disorder similar to alcoholic liver disease, but without alcohol consumption. The NAFLD spectrum ranges from simple steatosis to non-alcoholic steatohepatitis (NASH), cirrhosis, and hepatocellular carcinoma. ${ }^{2,3}$ Once cirrhosis is present, hepatocellular carcinoma may also develop. ${ }^{4}$ The early identification of fibrosis is important in children with NAFLD in order to prevent the development of liver disease in adulthood. The gold standard for diagnosis of liver fibrosis is a liver biopsy, an invasive procedure. Hence, less-invasive strategies are needed to identify liver fibrosis especially in children with NAFLD. These considerations are relevant for pediatric patients, in which liver biopsy generally has greater risks and is less acceptable than in adults. ${ }^{4}$

From the Department of Child Health, Medical School, Diponegoro University, Dr. Kariadi Hospital, Semarang, Indonesia.

Reprint requests to: Ninung RD Kusumawati, Department of Child Health, Medical School, Diponegoro University, Dr. Kariadi Hospital, Semarang, Indonesia. Tel. +62-24-8414296. E-mail: roseadhiani@yahoo. co.id 
One non-invasive procedure to diagnose liver fibrosis is the APRI. APRI is simple and less expensive than biopsy for detecting fibrotic processes in liver. We aimed to use APRI to detect fibrotic processes in obese children with NAFLD. The purpose of our study was to look for a correlation between APRI and BMI in obese children with fatty liver.

\section{Methods}

This cross-sectional study was conducted from August - September 2007 at a single junior high school in Semarang where the prevalence of obesity was $17.9 \% .{ }^{5}$ Obesity was defined as BMI at or above the 95th percentile, using CDC 2000 growth charts. Children with a history of alcohol consumption, jaundice after transfusion, or maternal hepatitis B, were excluded. Blood specimen examinations included complete blood count, cholesterol, and transaminase enzyme measurements. analysis and hypothesis test was used to analyze data. Correlations between BMI, weight and APRI were analyzed by Spearman's test due to the nonparametric data. Results were considered significant for $\mathrm{P}<0.05$.

\section{Results}

Of 37 obese children, we found 19 with bright liver by abdominal USG. There were 15 males and 4 females subjects. Their mean age was 13.2 years, ranging from 12.6 to 13.7 years. Subjects' characteristics are shown in Table 1.

The mean APRI of our subjects was 0.16 (SD 0.119), indicating that almost all subjects had no liver fibrosis. However, one patient with bright liver had APRI $>0.5$.

APRI and ALT had a significant positive correlation $(r=0.62)$. However, APRI was not significantly correlated with weight or BMI Table 2.

Table 1. Characteristics of subjects with bright liver

\begin{tabular}{lccc}
\hline Characteristics & $\mathrm{n}=19$ & Minimum & Maximum \\
\hline Mean age, years (SD) & $13.2(0.29)$ & 12.6 & 13.7 \\
Mean weight, $\mathrm{kg}(\mathrm{SD})$ & $75.5(10.74)$ & 53.8 & 101.8 \\
Mean BMI, $\mathrm{kg} / \mathrm{m}^{2}(\mathrm{SD})$ & $29.9(2.79)$ & 24.8 & 36.3 \\
Mean AST, IU/L (SD) & $28.9(22.61)$ & 12 & 118 \\
Mean ALT, IU/L (SD) & $49.2(60.74)$ & 15 & 291 \\
Mean platelets, $\mathrm{x} 10^{3} / \mathrm{mm}^{3}$ (SD) & $372.3(60.31)$ & 239 & 482 \\
Mean APRI, units (SD) & $0.16(0.119)$ & 0.07 & 0.63 \\
\hline AST : aspartate aminotransferase & $\mathrm{ALT}:$ alanine amino transferase & APRI : AST-platelet ratio index
\end{tabular}

APRI was calculated with formula : APRI $=$ [\{AST $($ IU/l) $/$ ALT_ULN $(I U / 1)\} \times 100] /$ platelet count. The value of AST was taken from subjects' measurements, while the upper limit of normal for ALT (ALT ULN) was a constant value, i.e., $50 \mathrm{IU} / \mathrm{L}$. An APRI $<0.5$ indicated no or minimal fibrosis, while APRI $>1.5$ indicated significant fibrosis. ${ }^{6}$

Abdominal USG was performed at Dr. Kariadi Hospital, Semarang, and assessed by a single radiologist. To reduce the intra-observer disagreement, the kappa value was assessed 1 month after the USG examinations. The kappa value of abdominal USG was 0.8.

All subjects' parents provided written informed consent. This study was approved by the Ethics Committee of Diponegoro University Faculty of Medicine/Dr. Kariadi Hospital, Semarang. Descriptive
Table 2. Correlation between weight, BMI, and ALT with APRI

\begin{tabular}{lcc}
\hline \multirow{2}{*}{ Variables } & \multicolumn{2}{c}{ APRI } \\
\cline { 2 - 3 } & $\mathrm{r}$ & $\mathrm{P}$ \\
\hline Weight & 0.33 & 0.166 \\
BMI & 0.35 & 0.145 \\
ALT & 0.62 & 0.004 \\
\hline BMI : body mass index & ALT : alanine amino transferase
\end{tabular}

\section{Discussion}

Stages of fibrosis may be assessed by the METAVIR scoring system. Scores are defined as: FO, no fibrosis; F1, portal fibrosis without septae; F2, portal fibrosis with rare septae; F3, numerous septae without cirrhosis; 
and F4, cirrhosis. ${ }^{6}$ Several non-invasive markers of liver fibrosis have been developed with the aim of discriminating between clinically insignificant (FO-F1) and significant fibrosis (> F2), as well as to identify established cirrhosis in patients with well-compensated chronic liver disease. Serum markers of hepatic fibrosis refer to the measurement of one or more molecules in blood or serum indicative of fibrosis in the liver. ${ }^{8}$ Proposed serum markers of hepatic fibrosis may be broadly categorized as either direct or indirect. Indirect markers reflect alterations in hepatic function, but do not directly reflect extracellular matrix (ECM) metabolism. Direct markers of liver fibrosis reflect the process of fibrogenesis, including ECM deposition or removal. ${ }^{7}$ Ideal serum markers would be liver specific, independent of metabolic alterations, easy to measure, minimally influenced by urinary and biliary excretion, reflective of fibrosis irrespective of cause, sensitive enough to discriminate between stages of fibrosis, and correlated with dynamic changes in fibrogenesis or fibrosis resolution. ${ }^{9}$ However, none of the available markers fulfill these ideal criteria. ${ }^{10}$

APRI is an indirect marker of liver fibrosis. Wai et al. found that the APRI was the simplest and the most accurate test for evaluation of two end points. ${ }^{11}$ APRI may be used to distinguish between those with and without significant fibrosis and cirrhosis, with negative predictive values of $86 \%$ and $98 \% .{ }^{11}$ We used APRI in our study because of its simplicity and availability in hospital settings, despite its lower sensitivity in predicting mild fibrosis.

The pathogenesis of NAFLD is not fully understood. Current theory suggests a "two hit" process. Lipid accumulation in hepatocytes results in macrovesicular steatosis. This accumulation process is a result of disorders in hepatic uptake, synthesis, degradation, and secretion of free fatty acids. These changes make the liver susceptible to a second "hit" which may result in inflammatory changes and disease worsening. Adiponectin and tumor necrosis factor (TNF)-alpha are fat-derived substances that regulate the hepatic inflammatory response. However, they are mutually antagonistic, inhibiting each other's production and activity. Adiponectin inhibits fatty acid uptake, stimulates fatty acid oxidation and lipid export, and enhances hepatic insulin sensitivity. TNFalpha recruits inflammatory cells to injured tissues and promotes insulin resistance. In patients with metabolic syndrome, cytokine imbalance may increase TNF-alpha production and reduce the activity of adiponectin. The combination of these conditions results in insulin resistance with fat accumulation, inflammation and cell death. ${ }^{12}$ Chronic hepatocellular death via necrosis and/or apoptosis initiates inflammatory responses and hepatic fibrogenesis. ${ }^{13}$ Hepatocellular regeneration in chronic liver injury is often inhibited due to the imbalance of growth factors and the distortion of liver architecture and circulation. Cellular debris and apoptotic bodies accumulate and initiate inflammatory responses, creating a self-amplifying loop that further compromises recovery from injury and facilitates the fibrogenic process. ${ }^{14}$ Subjects with visceral fat adiposity appear to be at risk for fatty liver due to their ability to transport free fatty acids directly into the portal vein for conversion to triglycerides within the liver. ${ }^{3}$

We found that there was no significant correlation between BMI and APRI. However, there was a significant correlation between weight and APRI. Although obesity is a NAFLD risk factor, we did not observe a correlation with liver fibrosis, represented by APRI. Perhaps APRI is less sensitive in predicting mild liver fibrosis, while NAFLD develops into liver fibrosis over a length of time. Our subjects may have been too young to have fibrosis. In contrast, Iacobellis et al. reported that BMI was an independent predictor of fibrosis (OR $=5.85,95 \%$ CI 1.6 to 21$)$ and their cutoff value for BMI was $26.3 \mathrm{~kg} / \mathrm{m}^{2}$. Their BMI evaluation showed a sensitivity and specificity of $66 \%$ and $71 \%$, respectively, in ascertaining the presence of fibrosis in children. ${ }^{15}$ In contrast with BMI and weight, AST and ALT had positive correlations with APRI, similar to a previous study that used AST and ALT as non-invasive predictors of liver fibrosis. ${ }^{16}$

In conclusion, we observed no correlation between BMI and APRI. However one obese subject $(1 / 37)$ with fatty liver had APRI $>0.5$, indicating liver fibrosis. Further study should be done with more subjects and using liver biopsy for confirmed diagnosis.

\section{References}

1. Mathur P, Das MK, Arora NK. Non-alcoholic fatty liver disease and childhood obesity. Indian J Pediatr. 2007;74:401-7. 
2. Nobili V, Alisi A, Vania A, Tiribelli C, Pietrobattista A, Bedogni G. The pediatric NAFLD fibrosis index: a predictor of liver fibrosis in children with non-alcoholic fatty liver disease. BMC Med. 2009; 7:21.

3. Karim MF, Al-Mahtab M, Rahman S, Khan M. NASH in children : a short review. Kust Med J. 2010;2:24-6.

4. Pacifico L, Poggiogalle E, Cantisani V, Menichini G, Ricci P, Ferraro F, et al. Pediatric nonalcoholic fatty liver disease: a clinical and laboratory challenge. World J Hepatol. 2010;2:275-88.

5. Mexitalia M. Faktor risiko obesitas pada remaja dikaji dari sudut energi expenditure dan polimorfisme gen uncoupling protein 2 dan 3 [dissertation]. [Semarang]: Diponegoro University; 2010.

6. Ashraf S. Non-invasive evaluation of liver fibrosis in patients with chronic hepatitis B and C [dissertation]. [Pakistan]: Baqai Medical University; 2006. Available from: http://prr. hec.gov.pk/Thesis/33S.pdf

7. Sebastiani G, Alberti A. Non-invasive fibrosis biomarkers reduce but not substitute the need for liver biopsy. World J Gastroenterol. 2006;12:3682-94.

8. Friedman SL. Liver fibrosis-from bench to bedside. J Hepatol. 2003;38:S38-53.
9. Kelleher TB, Afdhal N. Non-invasive assessment of liver fibrosis. Clin Liver Dis. 2005;9:667-83.

10. Afdhal NH, Nunes D. Evaluation of liver fibrosis: a concise review. Am J Gastroenterol. 2004;99:1160-74.

11. Wai CT, Greenson JK, Fontana RJ, Kalbfleisch JD, Marrero JA, Conjeevaram HS, et al. A simple noninvasive index can predict both significant fibrosis and cirrhosis in patients with chronic hepatitis C. Hepatology. 2003;38:518-26.

12. Hesham A-Kader H. Nonalcoholic fatty liver disease in children living in the obeseogenic society. World J Pediatr. 2009;5:245-54.

13. Rockey DC. Hepatic fibrosis, stellate cells, and portal hypertension. Clin Liver Dis. 2006;10:459-79.

14. Canbay A, Friedman S, Gores GJ. Apoptosis: the nexus of liver injury and fibrosis. Hepatology. 2004;39:273-78.

15. Iacobellis A, Marcellini M, Andriulli A, Perri F, Leandro G, Devito $\mathrm{R}$, et al. Non invasive evaluation of liver fibrosis in paediatric patients with nonalcoholic steatohepatitis. World J Gastroenterol. 2006;12:7821-5.

16. Siddiqi AI, Siddiqeh M, Mehmood A, Siddiqui AM. Alanin aminotransferase/aspartate aminotransferase ratio reversal and prolonged protrombin time: a specific indicator of hepatic cirrhosis. J Ayub Med Coll Abbottabad. 2007;19:22-4. 